\title{
Bilberry extract administration prevents retinal ganglion cell death in mice via the regulation of chaperone molecules under conditions of endoplasmic reticulum stress
}

\author{
This article was published in the following Dove Press journal: \\ Clinical Ophthalmology \\ I) October 2017 \\ Number of times this article has been viewed
}

\author{
Orie Nakamura' \\ Satoru Moritoh ${ }^{1,2}$ \\ Kota Sato ${ }^{1,3}$ \\ Shigeto Maekawa' \\ Namie Murayama' \\ Noriko Himori' \\ Kazuko Omodaka ${ }^{1,3}$ \\ Tetsuya Sogon ${ }^{4}$ \\ Toru Nakazawa ${ }^{1-3,5}$ \\ 'Department of Ophthalmology, \\ Tohoku University Graduate \\ School of Medicine, Miyagi, Japan \\ ${ }^{2}$ Department of Retinal Disease \\ Control, Tohoku University Graduate \\ School of Medicine, Miyagi, Japan; \\ ${ }^{3}$ Department of Ophthalmic Imaging \\ and Information Analytics, Tohoku \\ University Graduate School of \\ Medicine, Miyagi, Japan; ${ }^{4}$ R\&D \\ Department, Wakasa Seikatsu Co., \\ Ltd., Kyoto, Japan; ${ }^{5}$ Department of \\ Advanced Ophthalmic Medicine, \\ Tohoku University Graduate School \\ of Medicine, Miyagi, Japan
}

Purpose: To investigate the effect of bilberry extract anthocyanins on retinal ganglion cell (RGC) survival after optic nerve crush. Additionally, to determine details of the mechanism of the neuroprotective effect of bilberry extract anthocyanins and the involvement of endoplasmic reticulum stress suppression in the mouse retina.

Materials and methods: Anthocyanins in bilberry extract $(100 \mathrm{mg} / \mathrm{kg} / \mathrm{day}$ or $500 \mathrm{mg} / \mathrm{kg} / \mathrm{day})$ were administrated orally to $\mathrm{C} 57 \mathrm{BL} / 6 \mathrm{~J}$ mice. The expression levels of various molecular chaperones were assessed with quantitative reverse-transcription polymerase chain reaction, Western blotting, and immunohistochemistry. RGC survival was evaluated by measuring the gene expression of RGC markers and counting retrogradely labeled RGCs after optic nerve crush.

Results: The protein levels of Grp78 and Grp94 increased significantly in mice after bilberry extract administration. Increased Grp78 and Grp94 levels were detected in the inner nuclear layer and ganglion cell layer of the retina, surrounding the RGCs. Gene expression of Chop, Bax, and Atf4 increased in mice after optic nerve crush and decreased significantly after oral bilberry extract administration. RGC survival after nerve crush also increased with bilberry extract administration

Conclusion: These results indicate that oral bilberry extract administration suppresses RGC death. Bilberry extract administration increased Grp78 and Grp94 protein levels, an effect which may underlie the neuroprotective effect of bilberry extract after optic nerve crush. Thus, bilberry extract has a potential role in neuroprotective treatments for retinal injuries, such as those which occur in glaucoma.

Keywords: bilberry extract, molecular chaperones, ER stress, retinal ganglion cells, glaucoma

\section{Introduction}

Optic nerve injury, which occurs in optic atrophy and glaucoma, is a cause of irreversible visual loss. Diseases involving optic nerve injury are characterized by visual field loss, visual acuity loss, and the degeneration of the retinal ganglion cells (RGCs). ${ }^{1}$ The major risk factor for glaucoma is well known to be elevated intraocular pressure (IOP), but it is also recognized that some forms of glaucoma are multifactorial. Thus, to clarify the underlying pathological mechanisms of RGC death in optic nerve injury, several different animal models of axonal injury have been used. Previous studies, some by our own research group, have shown that oxidative stress and endoplasmic reticulum (ER) stress have critical roles in inducing the loss of RGCs. ${ }^{2-7}$
Correspondence: Kota Sato

Department of Ophthalmology, Tohoku University Graduate School of Medicine, I-I, Seiryo, Aoba, Sendai, Miyagi 980-8574, Japan

Tel +8I 227177294

Fax +81227177298

Email satokota@oph.med.tohoku.ac.jp 
These studies showed that anti-ER stress or/and anti-oxidative stress compound have potential to treat the degeneration of RGCs.

Cytotoxic stimulation such as hypoxia and elevation of calcium levels occurs during the accumulation of unfolded or misfolded protein, and then unfolded protein response (UPR) is induced. ${ }^{8}$ UPR is believed to be a protective mechanism through the elevation of ER chaperone to reduce misfolded protein. However, excess and prolonged ER stress promotes the signaling pathway of apoptosis. Previous studies have reported that the upregulation of chaperone molecules promotes cell survival during ER stress, in vitro and in vivo. In particular, Grp78, a chaperone molecule, has a critical role in suppressing apoptosis, ${ }^{9}$ and Grp94, another chaperone molecule, suppresses neuronal cell death during ischemia/reperfusion injury. ${ }^{10}$ These findings support the view that chaperone molecules such as Grp78 and Grp94 have an important role in protection of injured cells under ER stress.

Flavonoids are natural products which are widely distributed in vegetables and fruits and are well known to exert biological effects. ${ }^{11,12}$ Bilberry extract, which contains the flavonoid anthocyanin, has been shown to have a protective effect against cell damage caused by a variety of stress conditions. ${ }^{13-15}$ Therefore, daily consumption of flavonoidrich foods, such as bilberry extract, may have the potential to prevent various diseases. On the other hand, quercetin, a kind of flavonoid, also has an anti-apoptotic cell death effect during ER stress. ${ }^{16}$ However, it is unknown whether bilberry extract anthocyanin has an anti-ER stress effect directly.

In this study, we determined whether the oral administration of bilberry extract had a neuroprotective effect on the RGCs, and investigated the relationship of this effect with the activation of chaperone molecules that act against ER stress during optic nerve damage.

\section{Materials and methods Animals}

This study used 10 to 12-week-old male mice (C57BL/6J strain; SLC, Shizuoka, Japan). In total, 56 mice were used. For Western blotting, q-PCR, and immunohistochemical analysis, each experimental group included four mice. For retrograde labeling of RGCs, each experimental group included six mice. Maintenance and handling of the animals followed the guidelines of the Association for Research in Vision and Ophthalmology Statement for Use of Animals in Ophthalmic Vision Research, as well as the Tohoku University
Guidelines for Animal Research. The Ethics Committee for Animal Experiments at Tohoku University Graduate School of Medicine approved all experimental procedures.

\section{Bilberry extract administration}

Vaccinium myrtillus L. anthocyanoside (bilberry extract) (containing less than $36 \%$ anthocyanins) was provided by Wakasa Seikatsu Co., Ltd (Kyoto, Japan). The mice were divided into three groups: Group 1 received phosphatebuffered saline (PBS) as a vehicle, while Groups 2 and 3 received bilberry extract in doses of, respectively, 100 and $500 \mathrm{mg} / \mathrm{kg}$ of body weight/day, administered by oral gavage. Oral gavage was performed once a day for 3 days. Animals that also underwent optic nerve crush (NC) received daily oral gavage starting 3 days before $\mathrm{NC}$ and ending when the animals were sacrificed.

\section{Optic nerve injury}

Axonal injury was induced in the eyes of the mice as previously described. ${ }^{2,17}$ Briefly, the optic nerve was crushed $2 \mathrm{~mm}$ posterior to the globe with forceps for 10 seconds, while the animals were under deep anesthesia induced with ketamine $(100 \mathrm{mg} / \mathrm{kg})$ and xylazine $(9 \mathrm{mg} / \mathrm{kg})$. After the surgical procedure, antibiotic ointment was applied to the treated eyes.

\section{Retrograde labeling and counting of RGCs}

To determine the number of RGCs in the retinas of the mice, retrograde labeling was performed with 2\% Fluorogold (Fluorochrome, Denver, CO, USA) 7 days before optic nerve injury, as previously described. ${ }^{4}$ Seven days after optic nerve injury, the eyeballs were enucleated and fixed in 4\% paraformaldehyde (PFA) for $1 \mathrm{~h}$. Retinal flat mounts were then prepared on glass slides, as previously described. ${ }^{18}$ The average number of RGCs was then counted, as previously described. ${ }^{4}$

\section{Quantitative reverse-transcription polymerase chain reaction (qRT-PCR)}

Retinal samples from mice were collected 3 days after optic NC. Total RNA was extracted from the retinas, and cDNA was synthesized as previously described. ${ }^{19}$ qPCR was performed with a 7500 Fast Real-Time PCR system (Thermo Fisher Scientific, Waltham, MA, USA), as previously described. ${ }^{20}$ Predesigned TaqMan probes (Thermo Fisher Scientific) were used to detect the following genes: Grp78: Mm00517690_g1; Grp94: Mm00441926_m1; Chop: Mm00492097_m1; 
Bax: Mm00432051_m1; Atf4: Mm00515324_m1; Pou4f1: Mm02343791_m1; Pou4f2: Mm00454754_s1; Pou4f3: Mm04213795_s1; and Gapdh: Mm99999915_g1. Relative gene expression was calculated using the delta-delta Ct method, normalized to GAPDH mRNA.

\section{Western blotting}

Retinal proteins were extracted with RIPA buffer, and their concentrations were measured with a BCA assay (Thermo Fisher Scientific). Ten micrograms of mouse retinal proteins were separated in $10 \%$ polyacrylamide gel with SDS-PAGE and transferred onto an Immobilon-P membrane (MerckMillipore, Darmstudt, Germany). The membranes were blocked with 4\% block ACE (DS pharma biomedical, Osaka, Japan) and incubated with rabbit anti-Grp78 (Bip) (1:250, ab21685; Abcam, Cambridge, UK) or rat anti-Grp94 (1:250, ADI-SPA-850-F; Enzo Life Sciences, Exeter, UK) as the primary antibodies overnight at $4{ }^{\circ} \mathrm{C}$, and then incubated with HRP-conjugated secondary antibodies. Immunoblots were visualized with ECL prime detection reagent (GE Healthcare Bio-Sciences Corp., Piscataway, NJ, USA), and the immunoreactive bands were captured with ChemiDoc XRS (Bio-Rad Laboratories Inc., Hercules, CA, USA).

\section{Immunohistochemistry}

The eyes of the mice were perfused with 4\% PFA and cryosections were prepared, as previously described. ${ }^{4}$ The cryosections were washed in $0.05 \%$ Tween 20 in PBS (Tw-PBS) and incubated in blocking buffer (10\% donkey serum containing 2\% Tx-100 in PBS) for $30 \mathrm{~min}$ at room temperature. The cryosections were then incubated overnight at $4{ }^{\circ} \mathrm{C}$ in a blocking buffer containing rabbit anti-Grp 78 $(1: 100)$ or rat anti-Grp94 $(1: 100)$ as primary antibodies. After washing in Tw-PBS, the cryosections were then incubated with Alexa Fluor 488-conjugated donkey anti-rabbit or anti-rat antibodies (Thermo Fisher Scientific) for $1 \mathrm{~h}$ at room temperature. The sections were then mounted with Vectashield mounting medium containing DAPI (Vector Laboratories, Burlingame, CA, USA), and the fluorescence signal was captured with a fluorescence microscope (Axiovert 200; Carl Zeiss Meditec AG, Jena, Germany).

\section{Statistical analysis}

Statistical comparisons were made with an ANOVA followed by Dunnett's test to compare the mean in three groups and unpaired $t$-test to compare two samples. The significance level was set at $P<0.05$.

\section{Results \\ Bilberry extract administration up-regulated chaperone molecules in the mouse retinas}

The immunoblot analysis revealed the presence of Grp78 and Grp94 in the retinas 3 days after the administration of bilberry extract (Figure 1A). In the retinas of mice that received 100 or $500 \mathrm{mg} / \mathrm{kg} /$ day of bilberry extract, the protein level of Grp78 was $\sim 2.6$ fold and $\sim 3.2$ fold higher, respectively, than in the retinas of the PBS-treated control mice (Figure 1B). Additionally, the protein level of Grp94 was $\sim 1.4$ fold higher than in PBS-treated controls, a significant difference in the mice that received $500 \mathrm{mg} / \mathrm{kg} /$ day of bilberry extract (Figure 1C). However, the mRNA levels of Grp78 and Grp94 did not significantly differ in the mice that did or did not receive bilberry extract treatment (Figure 1D and E), suggesting that the upregulation of Grp78 and Grp94 does not depend on their transcriptional levels, and might be regulated during post-translational stabilization. To determine the type of cells that up-regulated the Grp78 and Grp94 proteins, we performed an immunohistochemical analysis targeting Grp78 and Grp94. Immunostaining with an anti-Grp78 antibody showed that the location of Grp78 was mainly surrounding the nuclei in the ganglion cell layer (GCL) and inner nuclear layer (INL) in PBS-treated mice. In a group of mice treated with $500 \mathrm{mg} / \mathrm{kg} /$ day of bilberry extract, Grp78 protein was mostly up-regulated in the GCL. After treatment with bilberry extract, Grp78 and Grp94 proteins were up-regulated in the GCL, suggesting that this treatment modulated the protein levels of Grp78 and Grp94 in the GCL specifically, and that bilberry treatment may provide pre-conditioning for RGC protection caused by ER stress. Grp78 and Grp94 protein was up-regulated mostly in the GCL, INL, and outer plexiform layer after the administration of 100 or $500 \mathrm{mg} / \mathrm{kg} /$ day bilberry extract for 3 days (Figure 2A and B). To determine potential side effects, we measured the body weight of the mice during treatment with bilberry extract. There were no significant changes in body weight between the vehicle-treated and bilberry-administered groups. Details of increases in body weight in each group are as follows: 3 days after PBS treatment, $0.6 \pm 0.5 \mathrm{~g}$; after $100 \mathrm{mg} / \mathrm{kg}$ bilberry treatment, $0.4 \pm 0.3 \mathrm{~g}$; and after $500 \mathrm{mg} / \mathrm{kg}$ bilberry treatment, $0.7 \pm 0.3 \mathrm{~g}$ (a one-way ANOVA showed no significant difference between the three groups; Table 1). No mice died during PBS or bilberry treatment. These results suggest that there were no side effects or toxicity issues associated with bilberry extract administration. 
A
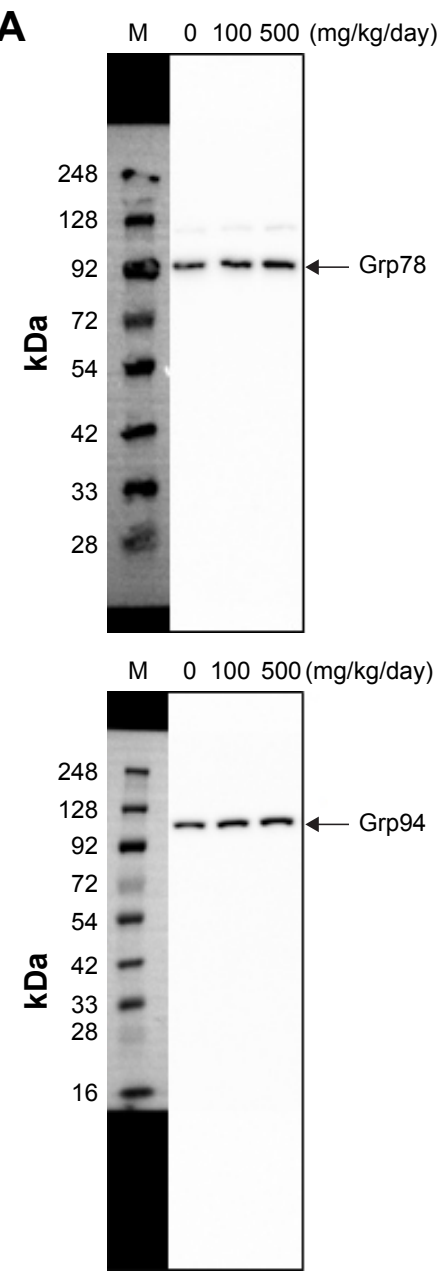

B

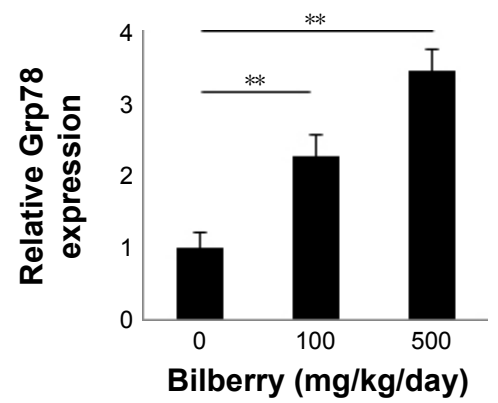

D

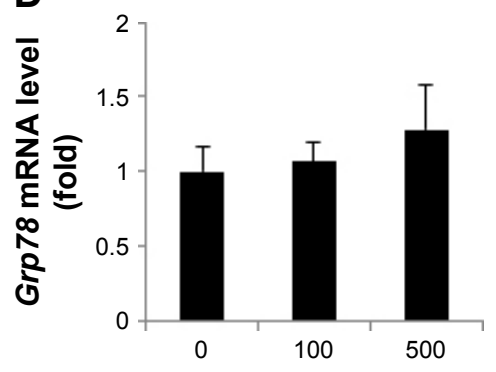

Bilberry (mg/kg/day)
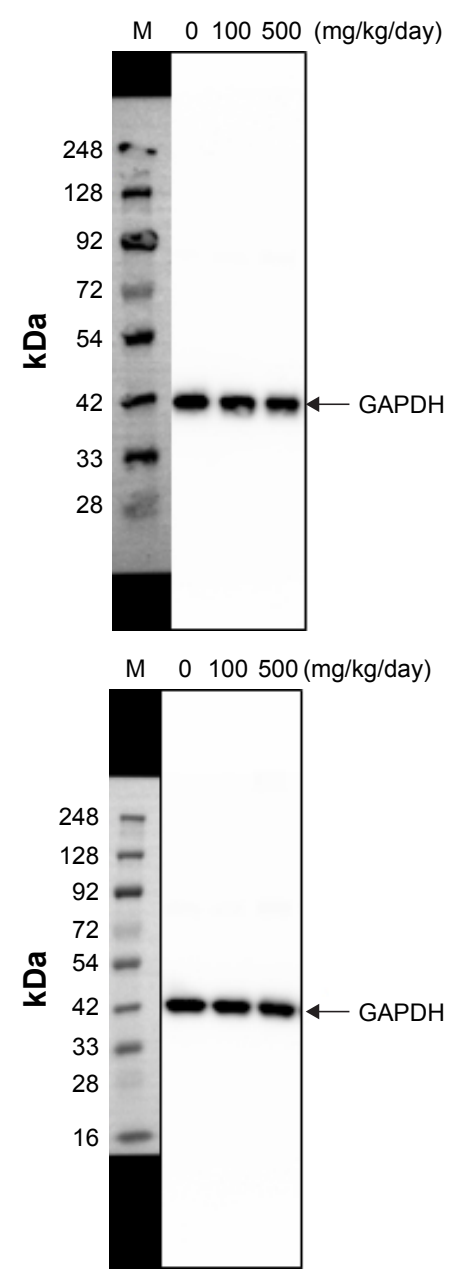

C
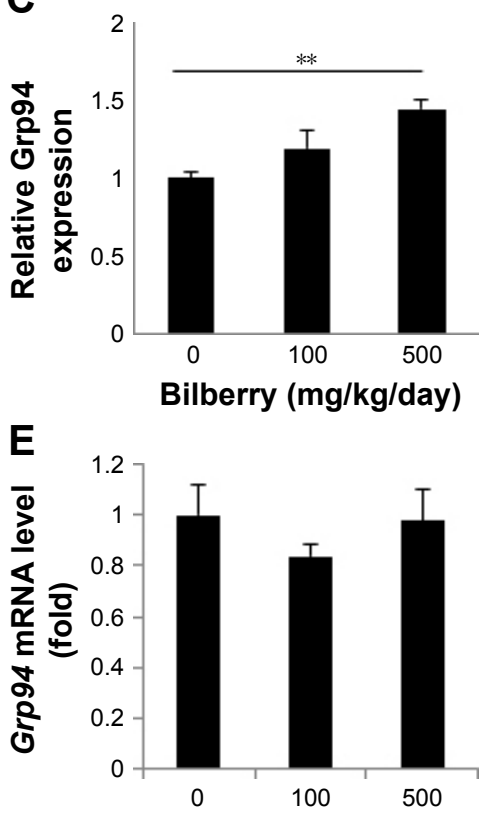

Bilberry (mg/kg/day)

Figure I Upregulation of chaperone molecules in the mouse retina 3 days after treatment with bilberry extract.

Notes: (A) Immunoblot analysis showing the protein expression of Grp78 and Grp94. GAPDH was used as a loading control. The relative expression levels of Grp78 (B) and Grp94 (C) were normalized to Gapdh, as shown in the histograms. Error bars show SD ( $\mathrm{n}=4$ ). The relative transcriptional expression of Grp78 (D) and Grp94 (E) was determined with qRT-PCR and was normalized to Gapdh. Error bars show SD $(n=4)$. **P<0.0I, Dunnett's post hoc comparison test.

Abbreviations: M, molecular marker; qRT-PCR, quantitative reverse-transcription polymerase chain reaction. 

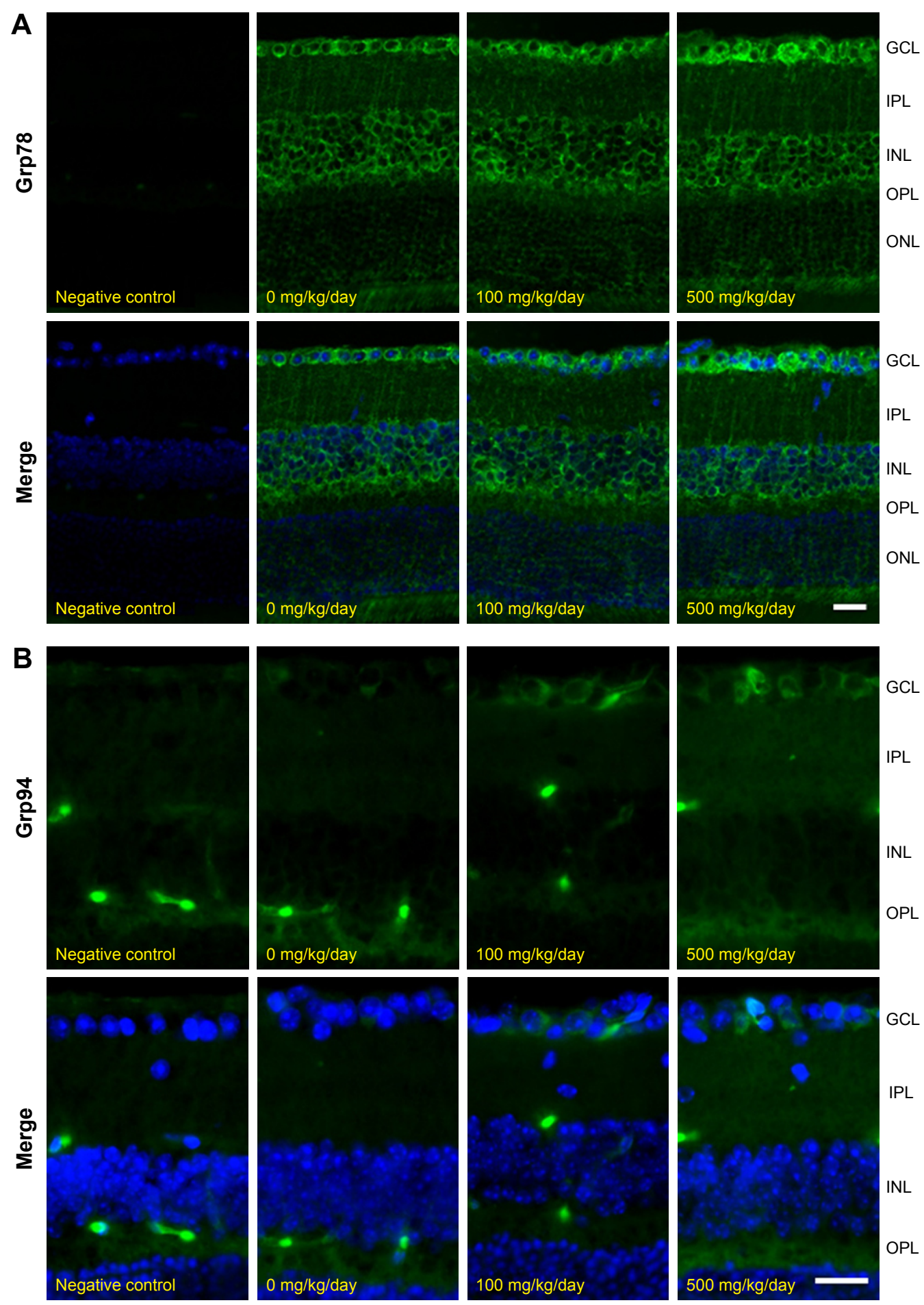

Figure $\mathbf{2}$ Localization of chaperone molecules in the mouse retina 3 days after treatment with bilberry extract.

Notes: Representative immunohistochemistry images showing the upregulation of Grp78 (A) and Grp94 (B) in the ganglion cell layer, inner nuclear layer, and outer plexiform later after treatment with bilberry extract. Scale bars: $20 \mu \mathrm{m}$.

Abbreviations: GCL, ganglion cell layer; IPL, inner plexiform layer; INL, inner nuclear layer; OPL, outer plexiform layer.

\section{Bilberry extract administration ameliorated the induction of ER stress and RGC degeneration}

After optic nerve injury, ER stress has been shown to induce progressive RGC death by activating apoptosis. ${ }^{21-23}$
Thus, this study evaluated the expression levels of genes involved in ER stress and apoptosis. Three days after optic NC, the gene expression levels of Chop and Bax were significantly higher ( $\sim 2.1$ fold and $\sim 2.0$ fold, respectively) than in animals that underwent a sham operation (Figure 3A). However, 
Table I Body weight measurement after bilberry extract administration

\begin{tabular}{llll}
\hline Oral treatment & $\begin{array}{l}\text { Body weight } \\
\text { (before } \\
\text { treatment) }\end{array}$ & $\begin{array}{l}\text { Body weight } \\
\text { (3 days after } \\
\text { treatment) }\end{array}$ & $\begin{array}{l}\text { Body weight } \\
\text { (increase) }\end{array}$ \\
\hline PBS as vehicle & $25.7 \pm 1.6 \mathrm{~g}$ & $26.3 \pm 1.6 \mathrm{~g}$ & $0.6 \pm 0.5 \mathrm{~g}$ \\
$100 \mathrm{mg} / \mathrm{kg}$ bilberry & $24.5 \pm 1.3 \mathrm{~g}$ & $25.0 \pm 1.4 \mathrm{~g}$ & $0.4 \pm 0.3 \mathrm{~g}$ \\
$500 \mathrm{mg} / \mathrm{kg}$ bilberry & $25.8 \pm 1.8 \mathrm{~g}$ & $26.3 \pm 1.6 \mathrm{~g}$ & $0.7 \pm 0.3 \mathrm{~g}$ \\
\hline
\end{tabular}

Note: Data presented as mean \pm standard deviation.

Abbreviation: PBS, phosphate-buffered saline.

bilberry extract administration significantly suppressed the induction of Chop, Bax, and Atf4 gene expression 3 days after optic NC, in comparison with mice that received only PBS (Figure 3A). In addition, the transcriptional level of Pou $4 f 2$ and Pou4f3, specific RGC markers, was higher ( $\sim 2.2$ fold and $\sim 2.3$ fold, respectively) in the retinas of mice that received bilberry extract than those of mice that received only PBS 3 days after optic NC (Figure 3B). Furthermore, the average number of retrogradely labeled RGCs in the retina was higher ( $\sim 1.2$ fold) after bilberry extract administration than after the administration of PBS 7 days after optic NC (Figure 4A and B).

\section{Discussion}

In this study, we showed that bilberry extract administration induced elevated levels of molecular chaperones in the retinas of mice. This induction of chaperone molecules might contribute to RGC protection by suppressing ER stress. This finding suggests that bilberry extract has a role in preventing ER stress and RGC death in the mouse retina after optic nerve injury. Previous work demonstrates that intravitreal injection of bilberry extract protects against excitotoxic damage induced by NMDA in the mouse retina. ${ }^{13}$ In addition, oral administration of bilberry extract ameliorates retinal damage caused by factors such as oxidative stress, photoreceptor damage, and the inflammatory response in the eyes of mice with endotoxin-induced uveitis. ${ }^{14}$ The novelty of the current study was its focus on the rescue of RGCs in an optic NC model, which is known to reflect glaucomatous damage in human subjects. Moreover, we investigated the oral administration of bilberry extract, to determine the potential of dietary supplementation with bilberry extract for glaucoma treatment. Previous work on the oral administration of bilberry extract
A

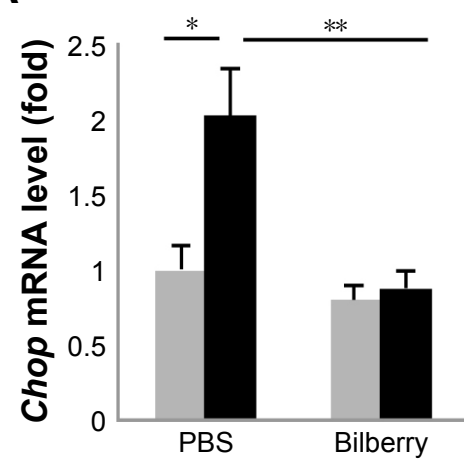

B

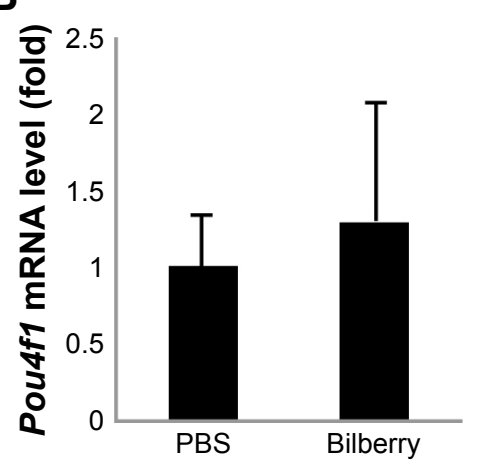

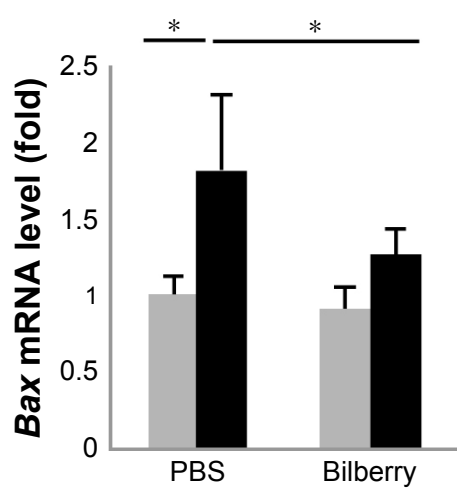

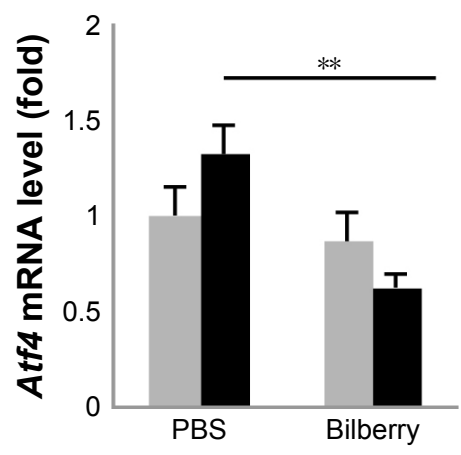

NT $\square \mathrm{NC}$
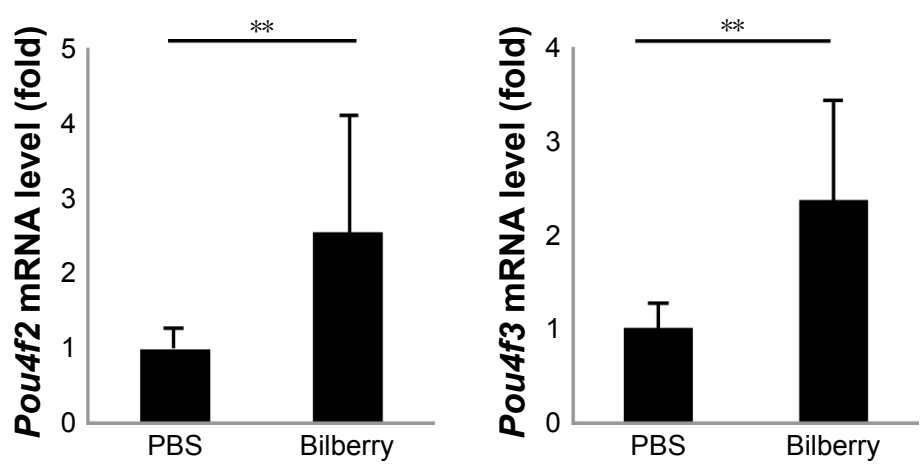

Figure 3 Gene expression of ER stress markers and RGC markers 3 days after optic nerve crush and treatment with bilberry extract (500 mg/kg/day). Notes: (A) The relative expression levels of Chop, Bax, and Atf4 mRNA were measured with qRT-PCR, normalized to Gapdh mRNA. (B) The relative expression levels of Pou4fl, Pou4f2, and Pou4f3 mRNA after optic nerve crush were measured with qRT-PCR, normalized to Gapdh. Error bars indicate $S D$ ( $n=4)$. $* P<0.05$, $* * P<0.01$, unpaired $t$-test. Abbreviations: ER, endoplasmic reticulum; NT, nontreated; NC, nerve crush; PBS, phosphate-buffered saline; qRT-PCR, quantitative reverse-transcription polymerase chain reaction; RGC, retinal ganglion cell. 
A

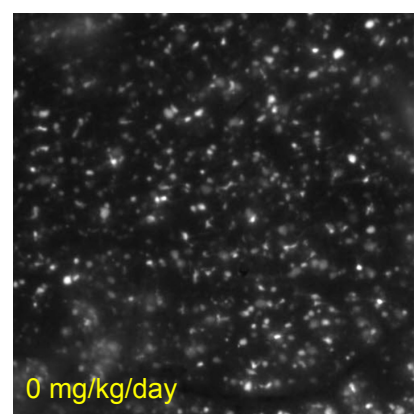

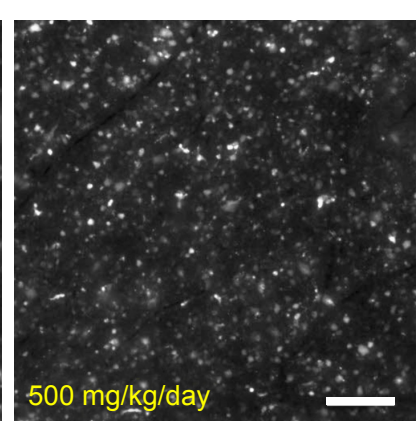

B

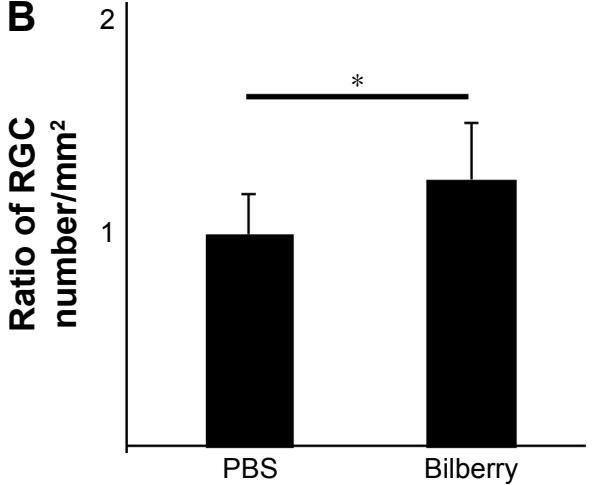

Figure 4 Prevention of RGC degeneration after treatment with bilberry extract ( $500 \mathrm{mg} / \mathrm{kg} /$ day).

Notes: (A) Representative image of retrogradely labeled RGCs 7 days after NC and the administration of PBS (left) or bilberry extract (right). Scale bar: I00 $\mu$ m (applies to both images). (B) Histogram showing the ratio of the average number of retrogradely labeled RGCs 7 days after NC and the administration of PBS (left) or bilberry extract (right). Error bars denote $S D(n=6) . * P<0.05$, unpaired $t$-test.

Abbreviations: NC, nerve crush; PBS, phosphate-buffered saline; RGC, retinal ganglion cell.

showed that it protected the gastric mucosa against ethanolinduced gastric ulcers by suppressing free radicals. ${ }^{24}$ Thus, taking into consideration previous work, our results may show that supplements containing bilberry extract can usefully protect against RGC damage in glaucoma, in addition to mitigating the inflammatory response and gastric ulcers.

Chaperone molecules are a class of proteins, the functions of which include the regulation of homeostasis and protection against ER stress. ${ }^{25}$ Among these molecules, Grp78 in particular has been shown to prevent ER stress-related cell death during the UPR. ${ }^{26}$ Previous work reported that Grp78 mediates CHOP suppression and reduces CHOP-dependent apoptosis. ${ }^{27}$ Furthermore, the PI3K/AKT pathway is activated by Grp78 on the cell surface. ${ }^{28}$ Additionally, glutamine metabolism, which contributes to cell survival, is promoted by c-Myc, which is induced by Grp78. ${ }^{29}$ These findings indicate that Grp78 acts to attenuate ER stress-dependent cell death and promote cell survival by facilitating the activation of several signaling and metabolism pathways. Grp94 is also induced by various stresses, including ER stress and glucose starvation. ${ }^{30}$ Adenovirus-mediated Grp94 overexpression suppresses neuronal cell death under oxidative stress and ischemic conditions, and perturbs calcium homeostasis..$^{10,31}$ In addition, silencing Grp94 with siRNA increases apoptosis induced by actinomycin D treatment in vitro, indicating that reduced Grp94 promotes apoptosis. ${ }^{32}$ In the field of ophthalmology, many studies have shown that optic nerve damage augments ER stress, as well as oxidative stress, in the RGCs, ${ }^{2-4,23}$ and drugs with anti-ER and anti-oxidative stress effects have been shown to reduce RGC loss., ${ }^{43-37}$ Therefore, our finding that bilberry extract administration increased the level of chaperone molecules, which are known to suppress ER stress, suggests that treatment with bilberry extract may be a promising way to prevent retinal and optic nerve damage in diseases such as glaucoma. We investigated Grp78 and Grp94 expression after optic NC in mice that received bilberry extract. We found that the protein level of Grp78 and Grp94 was slightly increased in the bilberry extract administration group 3 days after NC, similar to bilberry extract administration group without NC (Figure S1). However, the induction level of Grp78 and Grp94 protein in healthy mice was clearly higher than that in mice with NCinjured retinas. These findings suggest that pre-conditioning for Grp78 and Grp94 upregulation might be a crucial event in the mechanism of RGC protection after optic nerve injury in mice. However, detailed information on the mechanisms by which Grp78 and Grp94 modulate the cell survival pathway in the RGCs is unavailable at present, and needs to be elucidated in future studies.

In damaged neurons, unfolded proteins accumulate and induce cell death. In the retina of glaucoma mice model, after optic nerve injury, the ER stress pathway is activated and RGC death occurs. ${ }^{2,38}$ In Chop knockout mice, RGC death is reduced after optic NC. ${ }^{23}$ Thus, CHOP is considered a promising target for therapies to reduce RGC loss caused by ER stress after optic nerve injury. Atf4 is a transcriptional factor activated under ER stress and induces the expression of Chop, leading to ER stress-induced apoptosis. ${ }^{39}$ Our previous work suggested that the ATF4-CHOP pathway is the key upstream pathway inducing RGC loss during ER stress, which occurs in the early stages of axonal injury. ${ }^{2}$ In addition, prolonged ER stress promotes apoptosis via Bax activation and subsequent CHOP signaling. ${ }^{40}$ The current study obtained novel findings showing that bilberry extract administration suppressed the gene expression of Chop, Bax, and Atf4, suggesting that the mechanism of RGC preservation 
after the administration of bilberry extract anthocyanins may involve suppression of the CHOP pathway and modulation of the presence of chaperone molecules. Nevertheless, bilberry extract contains several kinds of anthocyanins, and while previous studies have demonstrated the anti-apoptotic effects of bilberry extract and/or its main anthocyanidin constituents (cyanidin, delphinidin, and malvidin), ${ }^{13}$ the current study could not identify the specific anthocyanin type that functions to elevate chaperone molecules in the RGCs. Thus, detailed information on the molecular mechanism underlying the regulation of chaperone molecule levels by bilberry extract remains unknown. This point awaits future investigation.

\section{Conclusion}

This study found that bilberry extract administration increased the levels of chaperone molecules in the mouse retina, and that bilberry extract administration prevented RGC loss after optic nerve injury. Furthermore, bilberry extract treatment prevented changes in the expression of genes related to ER stress. Therefore, these findings indicate that treatment with bilberry extract induces the expression of chaperone molecules, suppressing ER stress and preventing RGC loss.

\section{Acknowledgments}

We thank Mr Tim Hilts for editing this document and Ms Junko Sato for the technical assistance. This study was supported by grants from Wakasa Seikatsu Co., Ltd. and JSPS KAKENHI Grants-in-Aid for Scientific Research ([K Sato] 26893019).

\section{Author contributions}

O Nakamura and S Moritoh performed the experimental work. O Nakamura, S Moritoh, K Sato, S Maekawa, $\mathrm{N}$ Murayama, and T Nakazawa conceived and designed the experiments, analyzed the data, and provided advice on data interpretation. K Sato and T Nakazawa composed the manuscript. All authors contributed toward data analysis, drafting and critically revising the paper, gave final approval of the version to be published, and agree to be accountable for all aspects of the work.

\section{Disclosure}

This work was funded by Wakasa Seikatsu Co. Ltd. (T Sogon is an employee). The authors report no other conflicts of interest in this work.

\section{References}

1. Weinreb RN, Khaw PT. Primary open-angle glaucoma. Lancet. 2004; 363(9422):1711-1720.

2. Yasuda M, Tanaka Y, Ryu M, Tsuda S, Nakazawa T. RNA sequence reveals mouse retinal transcriptome changes early after axonal injury. PLoS One. 2014;9:e93258.

3. Himori N, Yamamoto K, Maruyama K, et al. Critical role of Nrf2 in oxidative stress-induced retinal ganglion cell death. JNeurochem. 2013; 127(5):669-680.

4. Yamamoto K, Maruyama K, Himori N, et al. The novel Rho kinase (ROCK) inhibitor K-115: a new candidate drug for neuroprotective treatment in glaucoma. Invest Ophthalmol Vis Sci. 2014;55(11): 7126-7136.

5. Yang L, Li S, Miao L, et al. Rescue of glaucomatous neurodegeneration by differentially modulating neuronal endoplasmic reticulum stress molecules. J Neurosci. 2016;36(21):5891-5903.

6. Ojino K, Shimazawa M, Izawa H, Nakano Y, Tsuruma K, Hara H. Involvement of endoplasmic reticulum stress in optic nerve degeneration after chronic high intraocular pressure in DBA/2J mice. J Neurosci Res. 2015;93(11):1675-1683.

7. Uchibayashi R, Tsuruma K, Inokuchi Y, Shimazawa M, Hara H. Involvement of Bid and caspase-2 in endoplasmic reticulum stress- and oxidative stress-induced retinal ganglion cell death. J Neurosci Res. 2011; 89(11):1783-1794.

8. Ron D, Walter P. Signal integration in the endoplasmic reticulum unfolded protein response. Nat Rev Mol Cell Biol. 2007;8(7):519-529.

9. Huang J, Chen $\mathrm{C}, \mathrm{Gu} \mathrm{H}$, et al. Mesencephalic astrocyte-derived neurotrophic factor reduces cell apoptosis via upregulating GRP78 in SH-SY5Y cells. Cell Biol Int. 2016;40(7):803-811.

10. Bando Y, Katayama T, Kasai K, Taniguchi M, Tamatani M, Tohyama M. GRP94 (94 kDa glucose-regulated protein) suppresses ischemic neuronal cell death against ischemia/reperfusion injury. Eur J Neurosci. 2003; 18(4):829-840.

11. Manganaris GA, Goulas V, Vicente AR, Terry LA. Berry antioxidants: small fruits providing large benefits. J Sci Food Agric. 2014;94(5): 825-833.

12. Sakakibara H, Ashida H, Kanazawa K. A novel method using 8-hydroperoxy-2'-deoxyguanosine formation for evaluating antioxidative potency. Free Radic Res. 2002;36(3):307-316.

13. Matsunaga N, Imai S, Inokuchi Y, et al. Bilberry and its main constituents have neuroprotective effects against retinal neuronal damage in vitro and in vivo. Mol Nutr Food Res. 2009;53(7):869-877.

14. Miyake S, Takahashi N, Sasaki M, Kobayashi S, Tsubota K, Ozawa Y. Vision preservation during retinal inflammation by anthocyanin-rich bilberry extract: cellular and molecular mechanism. Lab Invest. 2012; 92(1):102-109.

15. Ogawa K, Kuse Y, Tsuruma K, Kobayashi S, Shimazawa M, Hara H. Protective effects of bilberry and lingonberry extracts against blue light-emitting diode light-induced retinal photoreceptor cell damage in vvvvvvitro. BMC Complement Altern Med. 2014;14:120.

16. Park E, Chun HS. Protective effects of quercetin on dieldrin-induced endoplasmic reticulum stress and apoptosis in dopaminergic neuronal cells. Neuroreport. 2016;27(15):1140-1146.

17. Ryu M, Yasuda M, Shi D, et al. Critical role of calpain in axonal damage-induced retinal ganglion cell death. $J$ Neurosci Res. 2012; 90(4):802-815.

18. Kudo H, Nakazawa T, Shimura M, et al. Neuroprotective effect of latanoprost on rat retinal ganglion cells. Graefes Arch Clin Exp Ophthalmol. 2006;244(8):1003-1009.

19. Fujita K, Nishiguchi KM, Yokoyama Y, et al. In vivo cellular imaging of various stress/response pathways using AAV following axonal injury in mice. Sci Rep. 2015;5:18141.

20. Sato K, Nishiguchi KM, Maruyama K, et al. Topical ocular dexamethasone decreases intraocular pressure and body weight in rats. $J$ Negat Results Biomed. 2016;15:5. 
21. Puthalakath H, O'Reilly LA, Gunn P, et al. ER stress triggers apoptosis by activating BH3-only protein Bim. Cell. 2007;129(7): 1337-1349.

22. Song B, Scheuner D, Ron D, Pennathur S, Kaufman RJ. Chop deletion reduces oxidative stress, improves beta cell function, and promotes cell survival in multiple mouse models of diabetes. J Clin Invest. 2008; 118(10):3378-3389.

23. Hu Y, Park KK, Yang L, et al. Differential effects of unfolded protein response pathways on axon injury-induced death of retinal ganglion cells. Neuron. 2012;73(3):445-452.

24. Ogawa K, Oyagi A, Tanaka J, Kobayashi S, Hara H. The protective effect and action mechanism of Vaccinium myrtillus L. on gastric ulcer in mice. Phytother Res. 2011;25(8):1160-1165.

25. Hartl FU, Hayer-Hartl M. Molecular chaperones in the cytosol: from nascent chain to folded protein. Science. 2002;295(5561):1852-1858.

26. Flodby P, Li C, Liu Y, et al. The $78-\mathrm{kD}$ glucose-regulated protein regulates endoplasmic reticulum homeostasis and distal epithelial cell survival during lung development. Am J Respir Cell Mol Biol. 2016;55(1): 135-149.

27. Yan MM, Ni JD, Song D, Ding M, Huang J. Interplay between unfolded protein response and autophagy promotes tumor drug resistance. Oncol Lett. 2015;10(4):1959-1969.

28. Klionsky DJ. Autophagy revisited: a conversation with Christian de Duve. Autophagy. 2008;4(6):740-743.

29. Li Z, Wang Y, Wu H, Zhang L, Yang P, Li Z. GRP78 enhances the glutamine metabolism to support cell survival from glucose deficiency by modulating the beta-catenin signaling. Oncotarget. 2014;5(14): 5369-5380.

30. Kozutsumi Y, Segal M, Normington K, Gething MJ, Sambrook J. The presence of malfolded proteins in the endoplasmic reticulum signals the induction of glucose-regulated proteins. Nature. 1988;332(6163): $462-464$
31. Bando Y, Katayama T, Aleshin AN, Manabe T, Tohyama M. GRP94 reduces cell death in SH-SY5Y cells perturbated calcium homeostasis. Apoptosis. 2004;9(4):501-508.

32. Pan Z, Erkan M, Streit S, Friess H, Kleeff J. Silencing of GRP94 expression promotes apoptosis in pancreatic cancer cells. Int J Oncol. 2009;35(4):823-828.

33. Aydemir O, Naziroglu M, Celebi S, Yilmaz T, Kukner AS. Antioxidant effects of alpha-, gamma- and succinate-tocopherols in guinea pig retina during ischemia-reperfusion injury. Pathophysiology. 2004;11(3): $167-171$.

34. Tsuruma K, Shimazaki H, Nakashima K, et al. Annatto prevents retinal degeneration induced by endoplasmic reticulum stress in vitro and in vivo. Mol Nutr Food Res. 2012;56(5):713-724.

35. Himori N, Maruyama K, Yamamoto K, et al. Critical neuroprotective roles of heme oxygenase-1 induction against axonal injury-induced retinal ganglion cell death. J Neurosci Res. 2014;92(9):1134-1142.

36. Lee D, Shim MS, Kim KY, et al. Coenzyme Q10 inhibits glutamate excitotoxicity and oxidative stress-mediated mitochondrial alteration in a mouse model of glaucoma. Invest Ophthalmol Vis Sci. 2014;55(2): 993-1005.

37. Song W, Huang P, Zhang C. Neuroprotective therapies for glaucoma. Drug Des Devel Ther. 2015;9:1469-1479.

38. Yasuda M, Tanaka Y, Omodaka K, et al. Transcriptome profiling of the rat retina after optic nerve transection. Sci Rep. 2016;6:28736.

39. Oyadomari S, Mori M. Roles of CHOP/GADD153 in endoplasmic reticulum stress. Cell Death Differ. 2004;11(4):381-389.

40. Tabas I, Ron D. Integrating the mechanisms of apoptosis induced by endoplasmic reticulum stress. Nat Cell Biol. 2011;13(3):184-190. 


\section{Supplementary material}

A

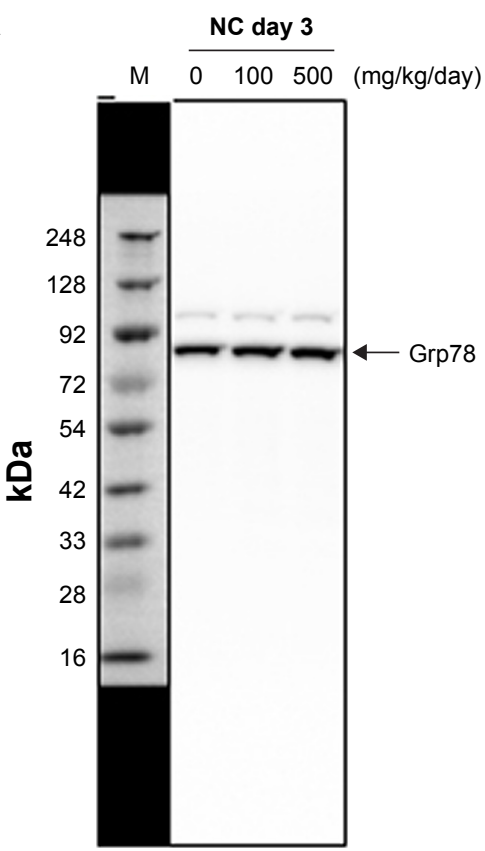

B

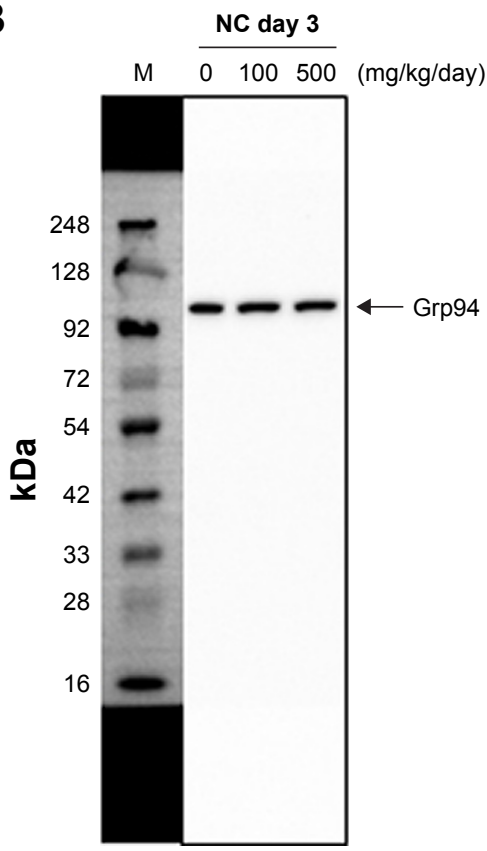

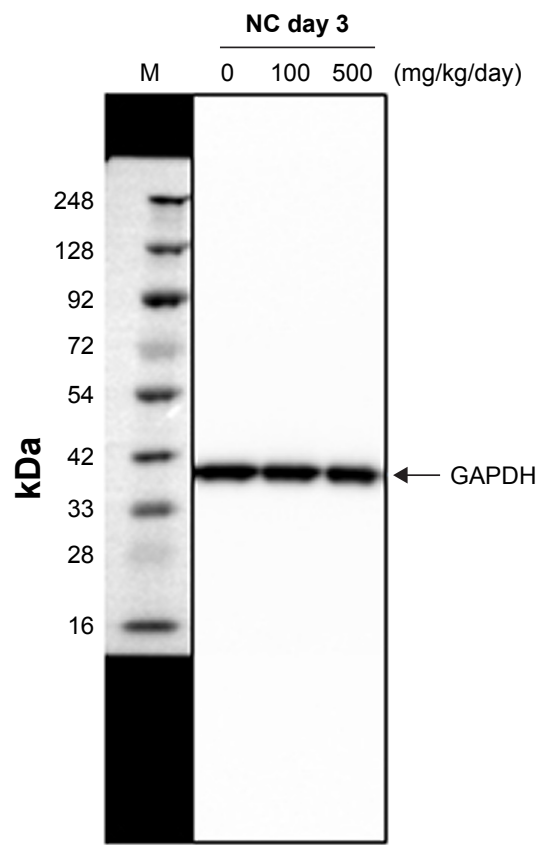

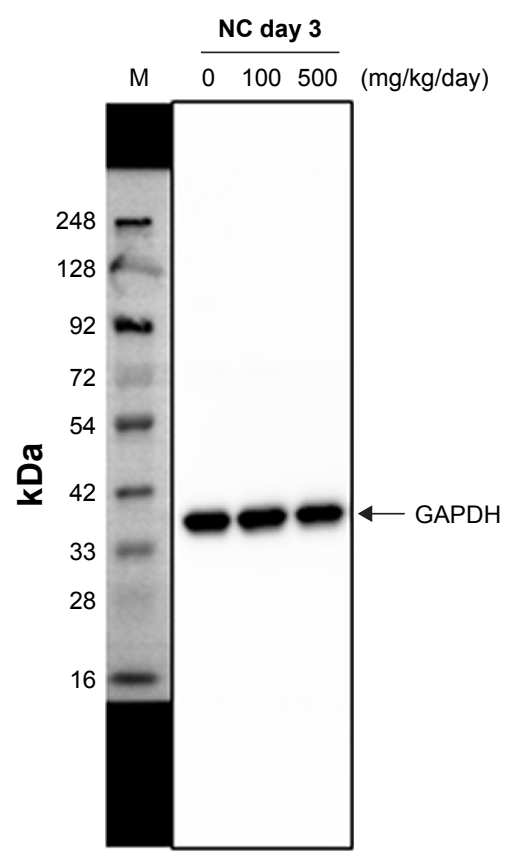

Figure SI Upregulation of chaperone molecules in the mouse retina 3 days after treatment with bilberry extract.

Notes: Immunoblot analysis showing the protein expression of Grp78 (A) and Grp94 (B). GAPDH was used as a loading control.

Abbreviations: M, molecular marker; NC, nerve crush.

Clinical Ophthalmology

\section{Publish your work in this journal}

Clinical Ophthalmology is an international, peer-reviewed journal covering all subspecialties within ophthalmology. Key topics include: Optometry; Visual science; Pharmacology and drug therapy in eye diseases; Basic Sciences; Primary and Secondary eye care; Patient Safety and Quality of Care Improvements. This journal is indexed on

\section{Dovepress}

PubMed Central and CAS, and is the official journal of The Society of Clinical Ophthalmology (SCO). The manuscript management system is completely online and includes a very quick and fair peer-review system, which is all easy to use. Visit http://www.dovepress.com/ testimonials.php to read real quotes from published authors. 